\title{
ANÁLISE DA EVOLUÇÃO DOS REGISTROS DO Lutzomyia longipalpis NO ESTADO DE SÃO PAULO AO LONGO DOS ANOS DE 1970-2014
}

\author{
ANALYSIS OF THE EVOLUTION OF Lutzomyia longipalpis RECORDS IN SÃO \\ PAULO STATE OVER THE YEARS 1970-2014
}

\author{
E. M. N. DE PAULA ${ }^{1}$, N. C. MARQUES ${ }^{2}$, M. B. D. OLIVARI ${ }^{2}$, B. F. IZOLA², J. H. B. TOSCANO², \\ A. P. R. GRISÓLIO², R. B. MEIRELLES-BARTOLI' ${ }^{1}$, A. A. B. CARVALHO²
}

\section{RESUMO}

A leishmaniose é uma zoonose de importância em saúde pública. Os vetores das leishmanioses são os flebotomíneos (Lutzomyia longipalpis). O comportamento alimentar do vetor favorece um maior contato vetor-hospedeiro, aumentando, assim, as chances do inseto tornar-se portador e transmitir a Leishmania. Trabalhos afirmam que o vetor foi detectado pela primeira vez em áreas urbanas no município de Araçatuba em 1977. E, a partir daí, pesquisas entomológicas dos anos seguintes já mostravam a presença do vetor em municípios vizinhos, mostrando que a disseminação do vetor foi rápida. O objetivo do presente trabalho foi analisar a evolução dos registro da presença do L. longipalpis no Estado de São Paulo (ESP) ao longo dos anos de 1970 (primeiro relato) a 2014 e a importância dessa análise.Trata-se de um estudo descrito utilizando-se dados referentes às pesquisas entomológicas realizadas pela Superintendência de Controle de Endemias (SUCEN) do ESP. Desde o ano de 1970, quando ocorreu o primeiro relato do L. longipalpis no ESP, em Araçatuba, até o ano de 2014, 171 municípios registraram a presença desse vetor em suas áreas. Os seis primeiros registros que ocorreram entre 1970 e 1995 são referentes às áreas rurais próximas a Araçatuba. De 1997 a 2014, L. longipalpis foi descrito em mais 164 municípios. Durante este período, entre 3 e 20 novos municípios por ano relataram a presença deste flebotomíneo, com destaque para o ano de 2012 em que houve 20 novos municípios e, mais de 63 relatando a presença do vetor nos últimos 5 anos. Uma observação relevante entre os achados é o fato de que 65 municípios possuíam somente o registro do L. longipalpis, ou seja, não possuem notificação de casos caninos ou humanos. As pesquisas entomológicas concluíram que os resgistros da presença do $L$. longipalpis veio aumentando consideravelmente e essas pesquisas são de extrema importância para o conhecimento de informações sobre a difusão e propagação da Leishmaniose, permitindo que os municípios utilizem estratégias eficientes de controle voltadas para o vetor com o objetivo de evitar casos humanos e caninos.

PALAVRAS-CHAVE: VETOR. LEISHMANIOSE VISCERAL. VIGILÂNCIA EPIDEMIOLÓGICA. SAÚDE PÚBLICA VETERINÁRIA.

AGRADECIMENTOS: Superintendência de Controle de Endemias (SUCEN) do Estado de São Paulo ÁREA TEMÁTICA: 6. Zoonoses

\footnotetext{
${ }^{1}$ Universidade Federal de Goiás (UFG), Regional Jataí, Unidade Jatobá, Laboratório de Sanidade Animal 2 Faculdade de Ciências Agrárias e Veterinárias da Universidade Estadual Paulista (UNESP) - Câmpus de Jaboticabal

* nat.cassaro@gmail.com
} 